\title{
Apply Fuzzy Seeking Control to High Precision Hard Disk Driver
}

\author{
Chin-Jou Liou, Sinn-Cheng Lin, Student Member, IEEE and Yung-Yaw Chen, Member,IEEE
}

Department of Electrical Engineering, Lab 202, National Taiwan University, Taipei, Taiwan, R.O.C

Tel: +886-02-3635251 ext. 202, Fax: +886-02-3671909, Email : cj@ipmc.ee.ntu.edu.tw

\begin{abstract}
This paper describes the application of fuzzy logic control on the track-seeking motion in the headpositioning servo mechanism of a hard disk. There are two major operations in the head-positioning servo of a hard disk, i.e. seeking and tracking. The seeking controller performs minimum-time movement of the read-write heads from their current track position to a target track specified by the file controller. The dynamic behaviors of the headpositioning servo is highly nonlinear, which make the derivations of an analytic model very difficult. Under the circumstances, the increasingly popular fuzzy logic control which does not require an exact mathematical model seems to be a good alternative in controller design. A fuzzy seeking controller is therefore designed and implemented on a high precision hard disk driver, the Zentek ZM3140. The proposed seeking controller can successfully move the read-write head servo mechanism to the desired target track. Our experimental results also show fast responses and robust behaviors of the servo system.
\end{abstract}

\section{Introduction}

The hard disk drive (HDD) should generally be compact, high-speed, and huge in storage density and capacity to satisfy requirements of modern personal computers and work stations. These are the reason that many researchers dedicated his time to HDD design to keep pace with host computers $[1]-[3],[6]-[7]$. A fuzzy seeking controller of the head-positioning servo mechanism in the ZM3140 HDD is designed in this paper. The purpose is hopefully to improve the access time of HDD. The head-positioning servo mechanism has two major functions; that is, track-seeking and track-following. The track-seeking function provides minimum time movement of the read-write heads from its current track to the target track. The track-following function maintains the position of the read-write heads exactly over the center of a given track with minimum displacement error in the presence of any possible disturbances. The average actuator access time

0-7803-2559-1/95 \$4.00 (C) 1995 IEEE is generically given by the sum of the average seek time and settling time. The value of average seek time for random seeks is found to be the time to seek to approximately one third of the tracks per recording band. How to dramatically reduce the average actuator seek time and how to effectively develop a control algorithm are therefore an important work of the research on the HDD.

Fuzzy control has been evolving into one of the most famous term in the word, and fuzzy control has been extensively spreading its power out in some scientific fields. A fuzzy logic controller (FLC) can be designed through means of a set of linguistic control rules related by a fuzzy implication rule of inference from a rough knowledge of the system dynamics and input-output relations. This FLC then convert the linguistic control strategy from experts or operator to an automatic control strategy for the controlled plant. An analytic model of the HDD is obtained relatively difficult as a consequence of nonlinear behaviors which are a unbalance in the magnetic circuit construction of the actuator, machining tolerances, and unevenness in magnetization of the magnet etc.. The controller design in traditional control methods requires a mathematical model of systems; such as, lead-lag frequency compensator, Hanselmann's LQG/LTR strategy [1], and Hasegawa's state feedback method [2] etc. . On the other hand, approximately reasoning-based fuzzy control which do not require analytic models have demonstrated a mass of successful application [4]. The FLC, moreover, not only both has applicability to a class of nonlinear systems but also ability to consider robustness issues for modeling uncertainties and disturbances.

The experimental HDD, ZM3140 3.5", 1" height, $120 \mathrm{MB}$, is supported by the Zentek Storage Inc., Shin-Chu, Taiwan with the specifications shown in Table 1. In this head-disk assembly (see Fig. 1 (a) and (b)), it is composed of the actuator, the amplifier, and the position error channel. The servo method is an embedded sector servo, in which the servo information is embedded in the data tracks as prerecorded sectors such that each head provide its own position information [5]. Fuzzy controller is 
constructed on a $80 \mathrm{C} 196 \mathrm{~KB}$ in circuit emulator (ICE). ICE is a emulation device of 80196 single chip to help the development of controller during the design period. Finally, the fuzzy seeking controller of HDD can precisely drive the read-write head servo mechanism to the desired track in the specified seek time without consuming too much control energy ,as demonstrated in experimental results.

\section{System description}

\subsection{System specifications}

The experimental HDD, ZM3140 3.5", I" height $120 \mathrm{MB}$ is supported by the Zentek Storage Inc., Shin-Chu, Taiwan with the specifications shown in table 2.1. In this head-disk assembly, it is embedded sector servo in which servo information is embedded in the data tracks as prerecorded sectors such that each head provide its own position information. Moreover it has 2 number of disks , 4 number of heads and 1540 number of data cylinders ( see Fig. 1 (a) and (b)).

\subsection{Head-Positioning Servomechanism}

The head-positioning servomechanism in a hard disk provides a method for locating a set of read-write heads in fixed radial locations over the disk surface and allows the repositioning of these heads from one radial location to another. A block diagram of a typical head-positioning servo with a linear voice-coil motor type, with two arms, 4 heads , and four disks is shown on Fig. 1 (b) From the block diagram, we see that the whole controlled plant is composed of the actuator, the amplifier that drives current into the coil, and the position error channel.

The servo system has two primary functions : one is to decide the position of the actuator and the other is to compare the difference of measured and desired position and decide a optimal control to reduce the error as soon as possible [5].

\subsection{Position error signal}

One of the significant parameters of HDD is the position error signal (PES), which is the output of the position error channel and proportional to the relative difference of the positions of the center of the servo head and the nearest track center. Hence the PES is period function of $x$ (radial position of the head) for stationary and ideal track centers ( see Fig. 2). The PES results from two sources of motion : motion of the actuator and motion of the disk surface itself. A simple mathematical description of the PES is given by ( refers to [5])

$$
P E S=k_{x} x_{e}\left\{M O D[(r-x)+c, w]-\frac{w}{2}\right\}
$$

where $w=$ track width

$x=$ radial position of the head

$r=$ track center position reference

0, for all $r, x$

$$
c=\text { any constant such that }(r(t)-x(t)+c)>
$$

\subsection{Actuator and Model}

As shown in Fig 1 (b), actuator prime mover is voice-coil motor (VCM) consisting of a permanent magnet structure and a movable bobbin or coil attached through a $\mathrm{T}$ plate to the comb of arms carrying the read-write heads. VCM have antecedents in the motors used in loudspeaker systems. A simple linear model for VCM is a second order system likewise $\frac{J}{s^{2}}$ where $\mathrm{J}$ is a gain constant through a system identification to identify its value. A procedure for identifying our HDD model is to use a white random signal through D/A converter into actuator to move the read -write head reach the target track and then reading the output of position error signal from A/D converter. A collection of input and output data pairs is used in analyzing frequency responses of the system for identifying the gain $J$. Notice that the gain $J$ is slight varying for different target track. Although FLC is need no any prescribe system model -- this is its main advantage, if we know a approximate rather than precise system model, it would greatly facilitate maters and look insight into the whole system under the development of FLC , whereas this dependency of operator and expert knowledge is also its drawback.

\section{Fuzzy Seeking Controller}

A PD-type FLC consists a set of rules, each rule can be symbolically described as follows:

if e is <property symbol> and $\Delta e$ is <property symbol> then $u$ is <property symbol>

where <property symbol> is the symbolic name of a linguistic value such as

if $e$ is positive big (PB) and $\Delta e$ is positive big $(P B)$ then $u$ is negative big (NB)

In Zentek HDD, the number of track in an individual disk surface is 1560 tracks. There are 256 units of division between two tracks. The read-write head seeks in if control force is positive, and vice versa. For the viewpoint of business, the $80 \mathrm{C} 196 \mathrm{~KB}$ single chip is used as a computational center of the controller. A look-up table technique is adopted for the purpose of achieving the real-time inference. But, making a trade-off between the resolution of 
the decision table and the size of the hardware memory is necessary for us. Therefore, a proper quantization of the universe of discourse is taken for the purpose of saving hardware memory and keeping the acceptable resolution. Three decision tables is constructed with different input variables under distinct circumstance. The inputs of our fuzzy seeking controller are $\{$ terr, tce $\}$ or $\{$ err_n, ce_n $\}$ depends on which look-up table we used, they are defined as follows:

$$
\begin{aligned}
& \text { terr(track err.) } \stackrel{\Delta}{=} \text { target track - current track } \\
& \text { err_n(absolute position err.) } \stackrel{\Delta}{=} \underset{\text { PES }}{ } * 256-\text { current }
\end{aligned}
$$

tce(track change of err.) $\stackrel{\Delta}{=}$ current terr - previous terr

ce_n (absolute position change of err.) $\triangleq$ tce*256

$\mathrm{x}($ absolute position $)=$ track number $* 256+$ PES.

Then three decision look-up tables is constructed as follows :

1st Table:

Input variable 1: terr,

$$
U . D .= \pm 600 \text { tracks and Q.L. }=12 \text { tracks }
$$

Input variable 2 : tce,

$$
U . D= \pm 10 \text { tracks and Q.L. }=1 \text { track }
$$

2nd Table:

Input variable 1: terr,

$$
\text { U.D. }= \pm 12 \text { tracks and Q.L. }=1 \text { track }
$$

Input variable 2: ce_n,

$$
\begin{gathered}
\text { U.D. }= \pm 256 \text { units of division and Q.L. }= \\
32 \text { units of division }
\end{gathered}
$$

3rd Table:

Input variable 1; err_n,

$$
\begin{gathered}
U . D .= \pm 256 \text { units of division and Q.L. }= \\
32 \text { units of division }
\end{gathered}
$$

Input variable 2: ce_n,

$$
\begin{gathered}
\text { U.D. }= \pm 16 \text { units of division and Q.L. }=1 \\
\text { unit of division }
\end{gathered}
$$

where U.D. means "universe of discourse" and Q.L. means "quantization level".

Notice that these look-up tables are actually generated from the same rule base with properly selecting the scaling factors to modify the universe of discourse. The membership functions of the term sets and the rule base are shown in Fig. 3 (a) and
Fig. 3 (b), respectively. The max-min inference engine and the center of gravity defuzzification method are adopted in this work..

\section{System Setup}

\subsection{System Setup}

The system setup is depicted in Fig. 4. The hard disk drive was placed on the shock-resistant pad to avoid unusual vibration. And the connection of devices was through RS232 cable to construct a closed control loop. Furthermore, the controlled plant was a voice-coil motor (VCM) which was not only driven by a VCM driver but also carry the read-write head servo mechanism for moving the read-write head to the desired target track as soon as possible. The input of controller was a position error signal (PES) with 220 millisecond sampling time from the position error channel, and the controller was then based on the PES to obtain the absolute position in the disk surface. The controller decided a control force to the VCM drive after comparison the discrepancy between the target track and a current track. The controller was constructed on $80 \mathrm{C} 196 \mathrm{~KB}$ in-circuit emulator (ICE) which is a device of emulating a $80 \mathrm{C} 196 \mathrm{~KB}$ single chip to assist the development of controller during the design period. The control programs was loaded into the Read Only Memory (ROM) rather than ICE when the controller design was accomplished; therefore, this control algorithm could implemented on commercial products.

\subsection{Experimental Apparatus}

The experimental apparatus was composed of the $80 \mathrm{C} 196 \mathrm{~KB}$ in circuit emulator, ZM3140 hard disk drive, power supply, 80486 personal computer, shock-resistant pad etc. The related devices are explained as follows:

\section{A. The $80 \mathrm{C} 196 \mathrm{~KB}$ microcontroller}

The MCS-96 family of 16-bit microcontrollers consists of two sub-families: the 8096 and the $80 \mathrm{C} 196$ microcontrollers. A $80 \mathrm{C} 196 \mathrm{~KB}$ microcontroller was chosen as a center of computations in this experiment.

\section{B. The $80 \mathrm{C} 196 \mathrm{~KB}$ in circuit emulator}

The $80 \mathrm{C} 196 \mathrm{~KB}$ In-Circuit Emulator (ICE) provided a means for developing, debugging and analyzing the software and hardware components of the $80 \mathrm{C} 196 \mathrm{~KB}$ microprocessor or microcontroller based systems. The model USP-96 was a second generation of Signum's 80C196 emulators (see Fig. 4.2). The emulation POD-196 KB/KC was plugged 
into the target processor socket, and was connected to ICE via a ribbon cable.

\section{Hard Disk Drive}

The experimental HDD was the Zentek ZM3140, as explained in section 2 system description.

\section{Shock-resistant pad}

The Shock-resistant pad was used to reduce possibly vibration by environment. This device was manufactured by Magfloat company in Japan with a total of $21 \mathrm{Kg}$ loaded capacity. But the HDD had only a weight of $0.5 \mathrm{Kg}$ (1.1 Pounds), a iron of $10 \mathrm{Kg}$ is added on the pad to obtain a higher sensitivity of this shock-resistant pad.

E. The 80486 personal computer

The 80486 personal computer (PC) was applied to development our fuzzy seeking control algorithms and executing ICE software so as to transfer the supremacy over the PC to the ICE.

\section{Experimental Results}

A read-write head moving from 1500th track to 1000 th track is taken into account in this present study. A step response has no overshoot, as demonstrated in Fig. 5 (a). The position error signal (PES), as observed from Fig. 5 (b), was getting to steady state in about $20 \mathrm{~ms}$. A state trajectory associated error with change of error in phase plane, as was illustrated in Fig. 5 (c). A control force driving the voice-coil motor was finally depicted in Fig. 5 (d). Some of advantages such as both the control effort in fuzzy logic seeking controller and the temperature on the HDD are less than those of the bang-bang control, as observed from a comparison with the bang- bang control [3]. This is one of reasons that inhere in fuzzy logic seeking control -- it is robust with a rejection from temperature and other disturbances.

\section{Conclusion}

We have implemented a fuzzy seeking controller on a high precision hard disk driver. The proposed controller can successfully and precisely drive the read-write head servo mechanism to the desired target track within about $20 \mathrm{~ms}$ without consuming too much control energy. To control a HDD by the fuzzy IF-THEN rules, it is not necessary to derive the mathematical model of HDD such that the controller should have the main advantages of the FLC, e.g., insensitivity, robustness, low-cost ... etc. However, it also has the shortcomings of the FLC, e.g., there is no systematic way for stability analysis. One of the future research topics is fo- cused on how to applied the fuzzy sliding mode control approach we had developed on the HDD.

\section{References}

[1] H. Hanselmann, "LQG-Control of a Highly Resonant Disk Drive Head Positioning Actuator", IEEE Trans. on Industry Electronics, Vol. 35, No. 1, Feb. 1988

[2] S. Hasegawa, "Fast Access Control of the Head Positioning", SPIE Proceedings. Vol. 12481990

[3] S. Youichida and Y. Kurita et. al. , "Quasifuzzy Inference Bang-Bang Access Servo ", IECON, pp. 1639-1644, 1991

[4] E. H. Mamdani, "Applications of Fuzzy algorithms for simple dynamic plant", Proc. IEE, vol. 121, no. 12 pp. $1585-1588,1974$

[5] R. Lawrence Comstock and Michael L. Workman, "Data Storage on Rigid Disks", IBM Co., Chap. 2.

[6] Chin-Jou Liou, Sinn-Cheng Lin, and Yung-Yaw Chen, " Fuzzy Seeking Control with an Application on High Precision Hard Disk Driver", International IEEE-IAS Industrial Automation and Control conference, Taipei, May 22-27, 1995

[7] Chin-Jou Liou, Sinn-Cheng Lin, and Yung-Yaw Chen, "Design seeking controller with FLC and FSMC for High Precision Hard Disk Driver", IASTED International Conference on Robotics and Manufacturing, Cancun, Mexico, June 14-16,19

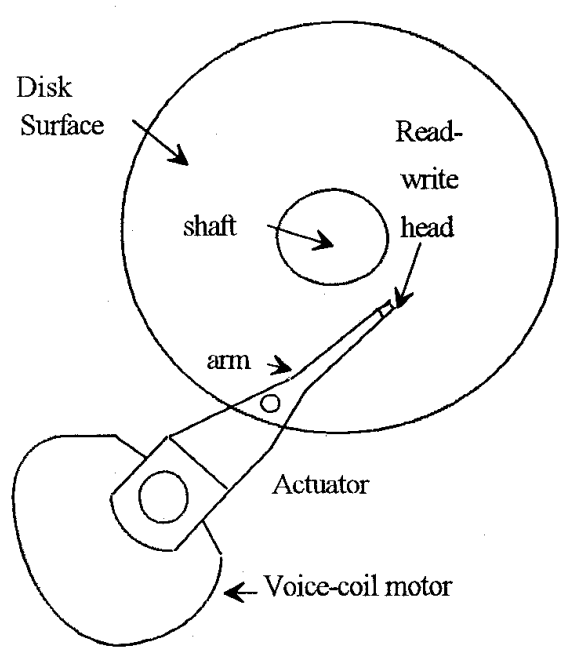

Fig. 1 (a) ZM3140 HDD configuration 


\begin{tabular}{|l|r|}
\hline Number of disks & 2 \\
\hline Number of heads & 4 \\
\hline Track density (TPI) & 1850 \\
\hline Bit density (BPI) & $36 \mathrm{k}$ \\
\hline Number of data cylinders & 1540 \\
\hline Number of data tracks & 6160 \\
\hline Sector size & 512 Bytes \\
\hline Sectors per track (Average) & 40 \\
\hline Disk rotation (RPM) & 3390 \\
\hline
\end{tabular}

Table 1 Specifications of ZM3140 3.5", 1" Height 120MB Hard Disk Driver

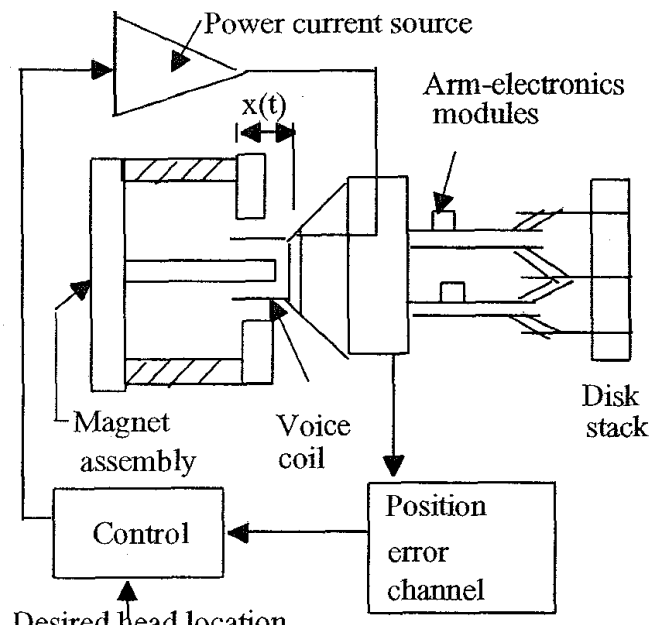

Fig. 1 (b) Block diagram of a typical head-positioning servo mechanism
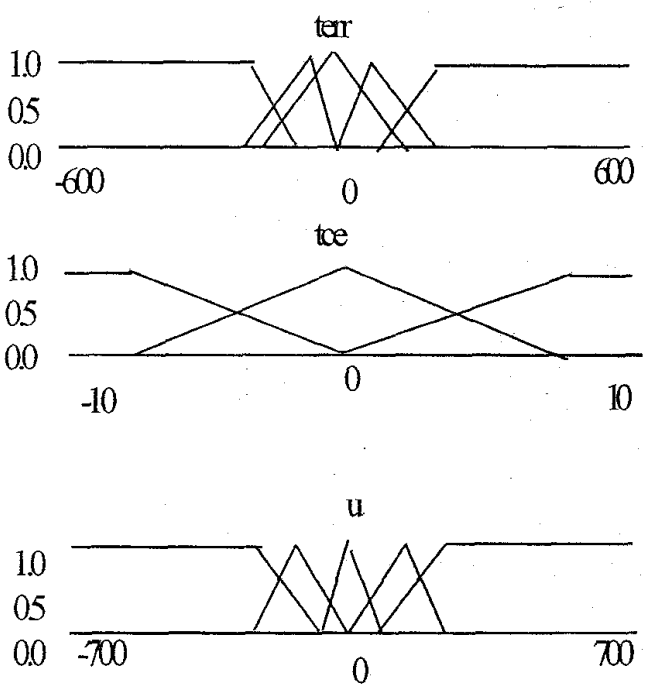

Fig. 3 (a) Membership functions

\begin{tabular}{|c|c|c|c|c|}
\hline \multirow{2}{*}{} & \multicolumn{3}{|c|}{ T CE } \\
\cline { 2 - 5 } & N B & Z E & P B \\
\hline T & N B & N B & N B & Z E \\
\cline { 2 - 5 } E & N S & N B & N S & P S \\
\cline { 2 - 5 } R R & Z E & N S & Z E & P S \\
\cline { 2 - 5 } & P S & N S & P S & P B \\
\hline & P B & Z E & P B & P B \\
\hline
\end{tabular}

Fig. 3 (b) Rule base

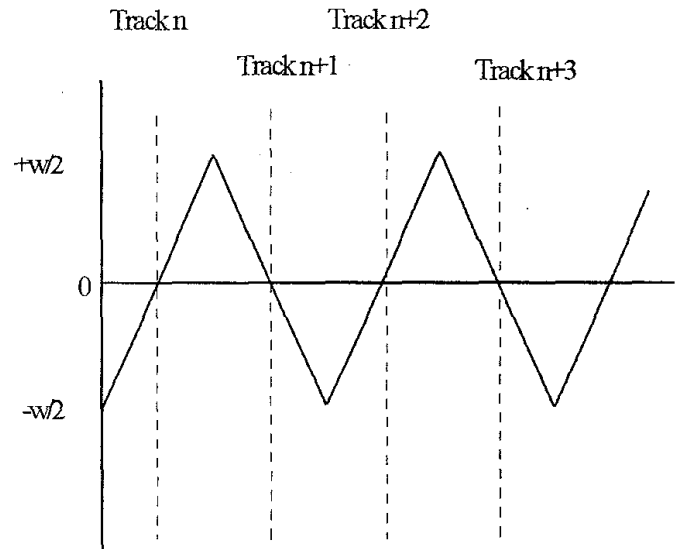

Fig. 2 A typical position error signal (PES)

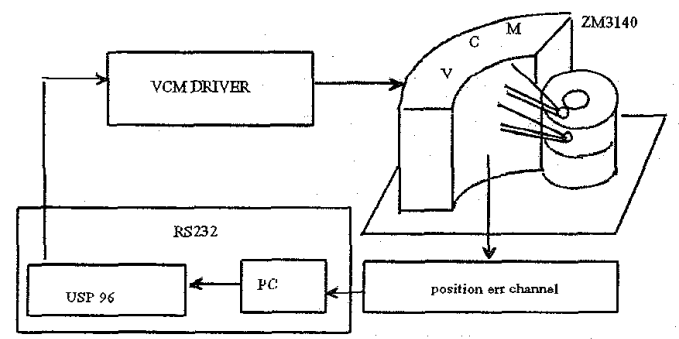

Fig. 4.1 Experimental apparatus and setup 


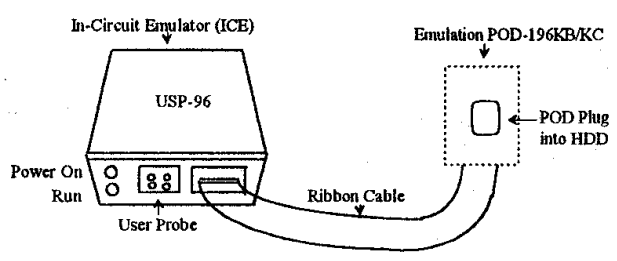

Fig. 4.2 The $80 \mathrm{C} 196$ Emulator and Emulation POD

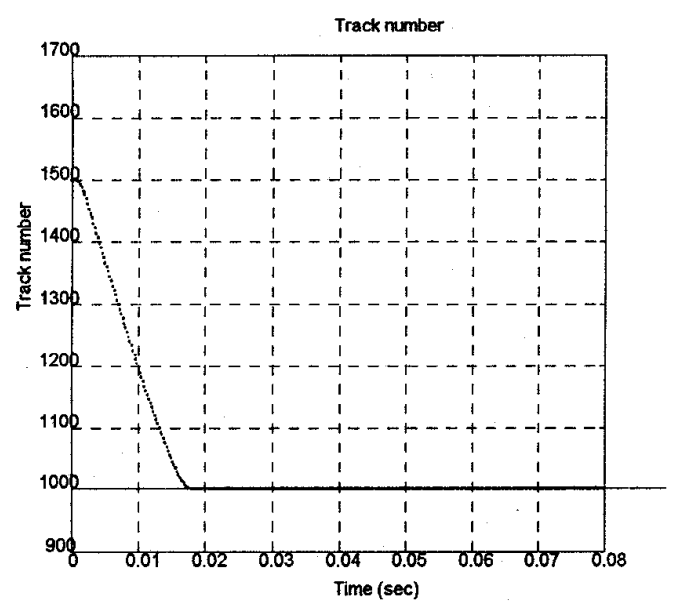

Fig. 5 (a) The step response of the track number

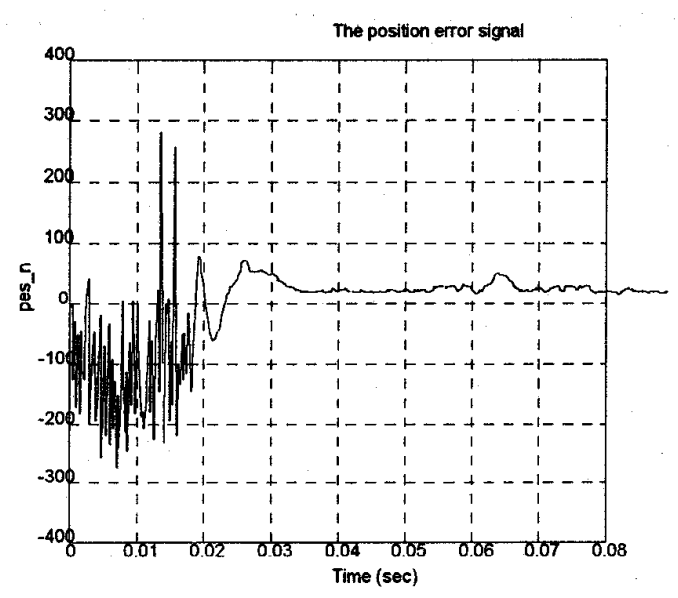

Fig. 5 (b) The position error signal

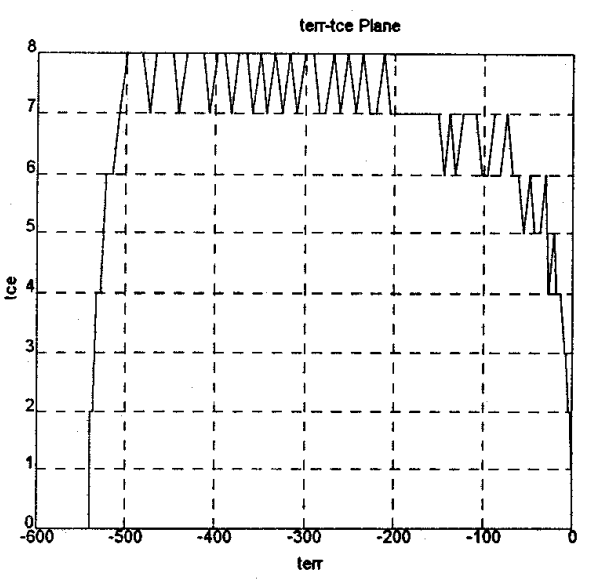

Fig. 5 (c) The phase plane of the state trajectory, error, e, and change of error, ce

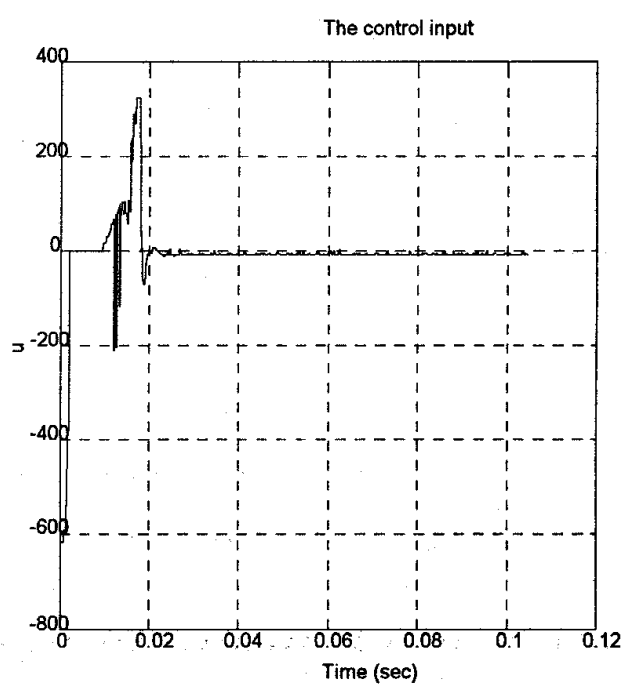

Fig. 5 (d) The control force, $u$ 